\title{
Antihyperglycemic Medications and Cardiovascular Risk Reduction
}

\author{
Sarah L Anderson and Joel C Marrs \\ Department of Clinical Pharmacy, University of Colorado Skaggs School of Pharmacy and Pharmaceutical Sciences, Aurora, Colorado, US
}

DOl: https://doi.org/10.17925/EE.2017.13.02.86

\section{Keywords}

Diabetes, cardiovascular disease, antihyperglycemic, glucagon-like peptide-1 receptor agonists (GLP-1) receptor agonists, sodium-glucose cotransporter-2 (SGLT2) inhibitors

Disclosure: Sarah L Anderson and Joel C Marrs have nothing to declare in relation to this article. No funding was received in the publication of this article. This study involves a review of the literature and did not involve any studies with human or animal subjects performed by any of the authors.

Authorship: All named authors meet the International Committee of Medical Journal Editors (ICMJE) criteria for authorship of this manuscript, take responsibility for the integrity of the work as a whole, and have given final approval to the version to be published.

Open Access: This article is published under the Creative Commons Attribution Noncommercial License, which permits any non-commercial use, distribution, adaptation and reproduction provided the original author(s) and source are given appropriate credit.

Received: 25 May 2017

Accepted: 13 July 2017

Citation: European Endocrinology, 2017;13(2):86-90

Corresponding Author: Sarah L Anderson;

Mail Stop C238, 12850 E. Montview Blvd.,

room V20-2129, Aurora, Colorado, 80045, US.

E: Sarah.Anderson@ucdenver.edu
Cardiovascular disease (CVD) is a common cause of morbidity and mortality in patients with type 2 diabetes (T2D). Patients with T2D have approximately twice the risk of developing CVD compared with the non-diabetic population, independent of other traditional CVD risk factors. ${ }^{1}$ Although the rate of death from CVD in patients with T2D is declining, it still exceeds that of the non-diabetic population. ${ }^{2}$ Improved glycaemia in patients with T2D may improve CVD and CVD-related death; however, it is still unclear whether optimal glycaemic control reduces the incidence of either of these. ${ }^{3}$ In several studies published during the past 10 years, improved glycaemic control and/ or addition of an antihyperglycaemic agent to a patient with T2D were associated with negative or neutral impact on cardiovascular (CV) outcomes. The Action to Control Cardiovascular Risk in Diabetes (ACCORD) trial demonstrated an increased risk of CV events and mortality in patients who received an intensive glucose-lowering intervention. ${ }^{4}$ The Rosiglitazone Evaluated for CV Outcomes in Oral Agent Combination Therapy for Type 2 Diabetes (RECORD) trial showed that the addition of rosiglitazone to therapy in patients with T2D increased the incidence of heart failure (HF). ${ }^{5}$ While the RECORD study was in progress, a meta-analysis of 42 trials involving patients with T2D who received rosiglitazone revealed an increased risk of myocardial infarction (MI) and CVrelated death in patients who received the drug. ${ }^{\circ}$

Despite the increased incidence of CVD and excess mortality attributed CVD-related deaths in the T2D population, it was not until these phase 4 studies unmasked a potential link between antihyperglycaemic agents and CV risk that the US Food and Drug Administration (FDA) required that new drug therapies for T2D undergo assessment of CV safety. The European Medicines Agency (EMA) followed with a similar requirement in $2012 .{ }^{\circ}$ In order to meet the FDA requirements, antihyperglycaemic CV outcomes trials in patients with T2D must include patients with advanced CVD, elderly patients and patients with renal dysfunction. These studies must track and include at least 24 months' CV safety data. Trials can be designed as non-inferiority or superiority and there are specific statistical requirements defining each trial type.?

Since this requirement to evaluate the CV safety of antihyperglycaemic agents was enacted, four studies have demonstrated that specific antihyperglycaemic agents (canagliflozin, empagliflozin, liraglutide and semaglutide) have beneficial effects on CV outcomes. Numerous other CV outcomes studies are ongoing with aspirations to demonstrate the same. In this review, we present the studies that have demonstrated a beneficial association between antihyperglycaemic agent use and CVD risk lowering, the ongoing CV outcomes trials with approved and investigational antihyperglycaemic agents, and reflect on the implications of these data for clinical practice.

\section{Positive cardiovascular outcome studies}

Positive CV risk-lowering findings in major clinical trials for antihyperglycaemic agents over the last two years have been essential to modifying the approach to glycaemic management and CV risk reduction therapy for patients with T2D. Sodium-glucose cotransporter-2 (SGLT2) inhibitors 
Table 1: Positive cardiovascular outcome trials

\begin{tabular}{|c|c|c|c|c|c|c|}
\hline Drug & Trial & $\mathrm{N}$ & Patient population & $\begin{array}{l}\text { Follow-up } \\
\text { median (years) }\end{array}$ & Primary outcome & Other outcomes \\
\hline Empagliflozin & $\begin{array}{l}\text { EMPA-REG } \\
\text { (NCT26378978) }\end{array}$ & 7,020 & T2D with established CVD & 3.1 & $\begin{array}{l}\text { MACE: } 10.5 \% \text { (E) VS. } 12.1 \%(P) \\
p<0.001\end{array}$ & $\begin{array}{l}\text { Death: } 8.3 \% \text { (E) vs. } 5.7 \%(P) ; p<0.001 \\
\text { CV Death: } 5.9 \% \text { (E) vs. } 3.7 \%(P) ; p<0.001 \\
\text { Hospitalisation for HF or CV death: } 8.5 \% \text { (E) } \\
\text { vs. } 5.7 \% \text { (P); } p<0.001\end{array}$ \\
\hline Liraglutide & $\begin{array}{l}\text { LEADER } \\
\text { (NCT27295427) }\end{array}$ & 9,340 & $\begin{array}{l}\text { T2D with high risk of CVD } \\
\text { or established CVD }\end{array}$ & 3.8 & $\begin{array}{l}\text { MACE: } 13.0 \%(L) \text { VS. } 14.9 \%(P) ; \\
p=0.01\end{array}$ & $\begin{array}{l}\text { Death: } 8.2 \% \text { (L) vs. } 9.6 \%(P) ; p=0.02 \\
\text { CV Death: } 4.7 \% \text { (L) vs. } 6.0 \%(P) ; p=0.007 \\
\text { Nephropathy: } 5.7 \% \text { (L) vs. } 7.2 \%(P) ; p=0.003\end{array}$ \\
\hline Semaglutide & $\begin{array}{l}\text { SUSTAIN-6 } \\
\text { (NCT27633186) }\end{array}$ & 2,735 & $\begin{array}{l}\text { T2D with high risk of CVD } \\
\text { or established CVD }\end{array}$ & 2.1 & $\begin{array}{l}\text { MACE: } 6.6 \%(S) \text { VS. } 8.9 \%(P) \\
p=0.02\end{array}$ & $\begin{array}{l}\text { Nonfatal stroke: } 1.6 \% \text { (S) vs. } 2.7 \%(P) ; p=0.04 \\
\text { Retinopathy: } 3.0 \% \text { (S) vs. } 1.8 \%(P) ; p=0.02 \\
\text { Nephropathy: } 3.8 \%(S) \text { vs. } 6.1 \%(P) ; p=0.005\end{array}$ \\
\hline Canagliflozin & $\begin{array}{l}\text { CANVAS } \\
\text { (NCT01032629) }\end{array}$ & 10,142 & $\begin{array}{l}\text { T2D with high risk of CVD } \\
\text { or established CVD }\end{array}$ & 2.4 & $\begin{array}{l}\text { MACE: } 26.9(C) \text { vs. } 31.5(P) \text { per } \\
1,000 \text { patient years, } p=0.02 \text { for } \\
\text { superiority }\end{array}$ & $\begin{array}{l}\text { Progression to albuminuria: } 89.4(\mathrm{C}) \mathrm{vs} .128 .7 \\
\text { (P) per } 1,000 \text { patient years (HR=0.73; } 95 \% \\
\mathrm{Cl} 0.67 \text { to } 0.79 \text { ) } \\
\text { Risk of amputation: } 6.3 \text { (C) vs. } 3.4(\mathrm{P}) \text { per } \\
\text { 1,000 Patient years (HR=1.97; } 95 \% \mathrm{Cl} 1.41 \\
\text { to } 2.75 \text { ) }\end{array}$ \\
\hline
\end{tabular}

$C=$ canagliflozin; $\mathrm{Cl}=$ confidence interval; $C V=$ cardiovascular; $C V D=$ cardiovascular disease; $E=$ emplagliflozin; $H F=$ heart failure; $H R=$ hazard ratio; $L=$ liraglutide; $M A C E=$ major adverse cardiovascular event; $P=$ placebo; $S=$ semaglutide; $T 2 D=$ type 2 diabetes .

(empagliflozin and canagliflozin) and glucagon-like peptide-1 receptor agonists (GLP-1 RA) (liraglutide and semaglutide) are the two major antihyperglycaemic classes to have demonstrated these positive CV findings to date (see Table 1).

Empagliflozin was the first of these four antihyperglycaemic agents to demonstrate positive CV outcomes in the Empagliflozin, Cardiovascular Outcomes, and Mortality in Type 2 Diabetes (EMPA-REG OUTCOME) trial. ${ }^{9}$ The EMPA-REG OUTCOME trial included 7,020 patients with T2D and established CVD. At baseline, patients had a mean age of 63 years, glycosylated haemoglobin (A1C) of $8.1 \%$, and more than half of patients had been diagnosed with T2D for more than 10 years. Approximately $75 \%$ of patients had coronary artery disease (CAD) and nearly 50\% had a history of MI. Patients were randomised to receive empagliflozin $10 \mathrm{mg}$ or $25 \mathrm{~m}$ g or placebo once daily. The primary composite major adverse cardiovascular event (MACE) included CV death, nonfatal MI, or nonfatal stroke. The study resulted in significant findings between the pooled empagliflozin- (10 $\mathrm{mg}$ and $25 \mathrm{mg}$ ) and the placebo-treated patients. The primary CV composite outcome occurred in 10.5\% and $12.1 \%$ of the empagliflozin- and placebo-treated patients, respectively, over a median of 3.1 years $(p=0.04)$. This results in a number needed to treat (NNT) of 65 patients with T2D and CVD over 3 years to prevent one additional major CV event. There was no difference between the rates of $\mathrm{Ml}$ or stroke between groups. However, patients treated with empagliflozin had a significantly lower rate of CV death $3.7 \%$ versus $5.9 \%$ ), all-cause death (5.7\% versus $8.3 \%$ ), and hospitalisation for heart failure (HHF) (5.7\% versus $8.5 \%$ ) compared to those treated with placebo $(p<0.001)$. These results support the rationale to optimise antihyperglycaemic therapy with empagliflozin in addition to first-line antihyperglycaemic therapy (e.g., metformin) in order to decrease the CV risk of patients with established CVD.

Liraglutide was the next agent to demonstrate positive CV outcomes in the Liraglutide Effect and Action in Diabetes: Evaluation of Cardiovascular Outcome Results (LEADER) trial..$^{10}$ The LEADER trial included 9,340 patients with T2D who were at least 50 years of age with established CVD or greater than 60 years of age with major CVD risk factors. Major CVD risk factors included: microalbuminuria or proteinuria, hypertension (HTN) and left ventricular hypertrophy (LVH), left ventricular systolic or diastolic dysfunction, or an ankle-brachial index (ABI) of $<0.9$. At baseline, enrolled patients had a mean age of 64 years, A1C of $8.7 \%$, and duration of diabetes of 13 years. Nearly all patients (81\%) had established CVD with prior MI and prior revascularisation being the most common diagnoses. Patients were randomised to receive $1.8 \mathrm{mg}$ of liraglutide or placebo once daily as a subcutaneous injection. The primary composite MACE was the same as EMPA-REG OUTCOME included CV death, nonfatal MI or nonfatal stroke. The study resulted in a significantly lower frequency of the composite outcome in the liraglutide-treated patients versus placebo. The primary CV composite outcome occurred in $13.0 \%$ and $14.9 \%$ of the liraglutide and placebo treated patients, respectively, over a median of 3.8 years $(p=0.01)$. This results in a NNT of 67 patients with T2D and CVD or CVD risk factors over 3 years to prevent one additional major $\mathrm{CV}$ event. There was no difference between the rates of nonfatal Ml, nonfatal stroke, or HHF between groups. Patients in the liraglutide group had statistically significantly lower rates of CV death (4.7\% versus $6.0 \% ; p=0.007$ ) and all-cause death $(8.2 \%$ versus $9.6 \% ; p=0.02$ ) compared to those receiving placebo. While there was a nonsignificant higher rate of pancreatitis in the placebo group compared to the liraglutide group (23 versus $18 ; p=0.44$ ), there was a signal toward increased risk of pancreatic cancer in the liraglutide-treated patients. There were 13 patients in the liraglutide group and five patients in the placebo group who developed a pancreatic carcinoma during the trial $(p=0.06)$. All 18 patients with pancreatic cancer passed away and it was discovered that another four patients in the placebo group (of a total of nine) passed away from 'malignancy related to pancreatic cancer'. The study authors concluded that due to small number of cases of pancreatic cancer, the association between liraglutide and pancreatic cancer risk in this study cohort could not be confirmed or excluded. In summary, these study results support the rationale to optimise antihyperglycaemic therapy with liraglutide in addition to first-line antihyperglycaemic therapy (e.g. metformin) in order to decrease the CV risk of patients aged 60 or greater with established CVD or patients 50 years of age or older with known CV risk factors.

Semaglutide was the third of these four antihyperglycaemic agents to demonstrate positive CV outcomes in the Trial to Evaluate Cardiovascular and Other Long-term Outcomes with Semaglutide in Subjects with Type 2 Diabetes (SUSTAIN-6). ${ }^{11}$ The SUSTAIN-6 trial included 2,735 patients with T2D who were at least 50 years of age, with established CVD, HF or chronic kidney disease (CKD) stage 3 to 5 , or who were 60 years of age 
or greater with major CV risk factors. Just as in the LEADER trial, major CVD risk factors included: microalbuminuria or proteinuria, HTN and $\mathrm{LVH}$, left ventricular systolic or diastolic dysfunction or an $\mathrm{ABI}$ of $<0.9$. At baseline, the mean patient age was 65 years, A1C was $8.7 \%$ and duration of diabetes was 14 years. Nearly all patients (92.8\%) had HTN, 60.5\% had ischaemic heart disease, $28.5 \%$ had moderate to severe renal impairment (estimated glomerular filtration rate [eGFR] $<60 \mathrm{ml} / \mathrm{min} / 1.73 \mathrm{~m}^{2}$ ), and 23.6\% had HF. Patients were randomised to receive a once-weekly subcutaneous injection of $0.5 \mathrm{mg}$ or $1 \mathrm{mg}$ of semaglutide or placebo. The primary composite MACE included CV death, nonfatal MI or nonfatal stroke. The study resulted in significant findings between the pooled semaglutide $(0.5 \mathrm{mg}$ and $1 \mathrm{mg}$ ) and the placebo-treated patients. The primary CV composite outcome occurred in $6.6 \%$ and $8.9 \%$ of the semaglutide- and placebo-treated patients, respectively, over a median of 2.1 years $(p<0.001)$. This results in a NNT of 31 patients with T2D and CVD or CVD risk factors over 3 years to prevent one additional major $\mathrm{CV}$ event. No difference was noted between the rates of nonfatal MI between groups, but patients treated with semaglutide experienced a significantly lower rate of nonfatal stroke ( $1.6 \%$ versus $2.7 \%$; $p=0.04$ ) and revascularisation (5.0\% versus $7.6 \% ; \mathrm{p}=0.003$ ) compared to those treated with placebo. Diabetic retinopathy occurred more frequently in semaglutide-treated patients compared to those who received placebo (3.0\% versus 1.8\%; hazard ratio $[\mathrm{HR}]=1.76$; $95 \%$ confidence interval $[\mathrm{Cl}]$, 1.11 to $2.78 ; \mathrm{p}=0.02$ ). The number needed to harm (NNH) is 58 patients over 3 years to see one additional case of diabetic retinopathy. It should be noted, however, that $83.5 \%$ of patients who developed a retinopathyrelated complication during the study had pre-existing retinopathy at baseline. These study findings support the rationale to optimise antihyperglycaemic therapy with semaglutide in addition to first-line antihyperglycaemic therapy (e.g., metformin) in order to decrease the CV risk of patients with established CVD with or without CKD who do not have evidence of diabetic retinopathy.

Most recently, canagliflozin has become the fourth antihyperglycaemic agent to demonstrate positive CV outcomes, albeit at an increased risk of limb or digit amputation. The CANagliflozin cardioVascular Assessment Study (CANVAS) trial evaluated the risk for MACE in patients with T2D and high CVD risk or existing CVD who received either canagliflozin $100 \mathrm{mg}$ or $300 \mathrm{mg}$ daily) or placebo. Data from CANVAS were combined with data from the Study of the Effects of Canagliflozin on Renal Endpoints in Adult Subjects with Type 2 Diabetes Mellitus [CANVAS-R] in a prespecified integrated analysis of CV safety outcomes. Patients enrolled in CANVAS-R received canagliflozin $100 \mathrm{mg}$ daily or placebo. The CANVAS programme (CANVAS and CANVAS-R) included 10,142 patients with T2D and at high risk for or existing CVD. Patients enrolled had a mean age of 63.3 years, baseline A1C of $8.2 \%$, and duration of diabetes of 13.5 years. ${ }^{12}$ A majority of patients (65.6\%) had existing CVD. The primary composite MACE included CV death, nonfatal MI or nonfatal stroke. The CANVAS program resulted in significant findings between the canagliflozin and the placebo-treated patients. The primary CV composite outcome occurred in 26.9 and 31.5 participants per 1,000 patient-years in the canagliflozin and placebo treated patients, respectively, over a median of 2.4 years ( $p=0.02$ for superiority). This results in a number needed to treat (NNT) of 179 patients over 3 years to prevent one additional major CV event. Progression to albuminuria was less frequent in the canagliflozin-treated patients compared with those in the placebo group (89.4 versus 128.7 events per 1,000 patient-years; $\mathrm{HR}=0.73 ; 95 \% \mathrm{Cl}, 0.67$ to 0.79 ). Serious adverse events were less frequent in the canagliflozin group compared to placebo (104.3 versus 120.0 patients with an event per 1,000 patientyears; $\mathrm{HR}=0.93 ; 95 \% \mathrm{Cl}, 0.87$ to 1.00 ). However, there was a significantly higher risk of amputation in the canagliflozin group compared to placebo
(6.3 versus 3.4 participants with amputation per 1,000 patient-years; $\mathrm{HR}=1.97 ; 95 \% \mathrm{Cl}, 1.41$ to 2.75$)$. This corresponds to a number needed to harm (NNH) of 277 patients over 3 years to see one additional amputation. Most affected patients (71\%) had a toe or metatarsal amputation and risk of amputation was highest among those with a history of amputation or concomitant peripheral vascular disease (PVD). Study authors acknowledge that the increased risk of amputation is a new finding and caution should be exercised when using canagliflozin in patients at risk for amputation. The US prescribing information for canagliflozin has been updated to include a new black box warning regarding the risk of amputation. Overall, these study findings support the rationale to optimise antihyperglycaemic therapy with canagliflozin in addition to first line antihyperglycaemic therapy (e.g. metformin) in order to decrease the $\mathrm{CV}$ risk of patients at high risk for or with established CVD. Healthcare providers should screen patients for risk factors for amputation (e.g., PVD) prior to prescribing canagliflozin. Patients and healthcare providers will need to weigh the $\mathrm{CV}$ benefit against the risk of amputation when considering canagliflozin for the treatment of T2D.

In addition to these individual drug studies, the Comparative Effectiveness of Cardiovascular Outcomes in New Users of SGLT-2 Inhibitors (CVDREAL) trial was a large, real-world study that retrospectively evaluated the risk for HHF, all-cause mortality, and the combined endpoint of HHF or all-cause mortality in patients with T2D (with and without CVD) who were new users of an approved SGLT2 inhibitor (canagliflozin, dapagliflozin and empagliflozin) compared to patients with T2D who were new users of any other antihyperglycaemic agent. ${ }^{13}$ Safety outcomes were not assessed. Data was collected via medical claims, primary care or hospital records, and national registries for nearly 310,000 patients with T2D across six countries. Patients newly initiated on an SGLT2 inhibitor were propensity score-matched with patients newly initiated on any other antihyperglycaemic agent (including fixed-dose combinations). After matching, the mean age was 57 years and 13\% had CVD. Baseline A1C was not reported. Most new users of SGLT2 inhibitors received either canagliflozin (51\%) or dapagliflozin (46\%). Insulin was the most common index antihyperglycaemic agent (34\%) for patients not on an SGLT2 inhibitor, followed by dipeptidyl peptidase-4 (DPP-4) inhibitors (18\%). Data analysis demonstrated that SGLT2 inhibitors reduce risk of $\mathrm{HHF}$ by $39 \%$ and all-cause mortality by $51 \%$. The study authors concluded that these data confirmed the positive outcomes demonstrate in EMPAREG OUTCOME and further support that CV benefit may be a class effect with SGLT2 inhibitors.

\section{Ongoing or unpublished cardiovascular outcome studies}

In addition to EMPA-REG OUTCOME, LEADER, SUSTAIN-6 and the CANVAS programme, multiple other studies evaluating the CV outcomes of individual antihyperglycaemic agents or classes of agents are currently ongoing or recently completed but not yet published (see Table 2). Cardiovascular outcomes trials of GLP-1 RAS, DPP-4 inhibitors, and SGLT2 inhibitors have either recently been completed or are scheduled to be completed within the next two years and will provide additional evidence that will undoubtedly influence antihyperglycaemic selection for patients with T2D and established CVD or at high risk of CVD.

There are five GLP-1 RA studies either recently completed or due to be completed. The studies are diverse in their methods and outcomes; if the $\mathrm{CV}$ trial data are consistently favourable, this will strengthen the case that beneficial CV effect is a class effect. However, if the trial data are neutral or negative, this could complicate the role in therapy of GLP-1 RAS. A Study to Evaluate Cardiovascular Outcomes in Patients with Type 2 Diabetes 
Table 2: Summary of ongoing or unpublished cardiovascular outcome trials

\begin{tabular}{|c|c|c|c|c|c|}
\hline Drug & Trial & $\mathrm{N}$ & Intervention & Comments & Completion date \\
\hline Exenatide in DUROS & $\begin{array}{l}\text { FREEDOM-CVO } \\
\text { (NCT01455896) }\end{array}$ & 4,000 & $\begin{array}{l}\text { Exenatide in DUROS } 60 \text { mcg daily } \\
\text { versus placebo }\end{array}$ & Drug not yet FDA or EMA approved & March 2016 \\
\hline Liraglutide & $\begin{array}{l}\text { MAGNA VICTORIA } \\
\text { (NCT01761318) }\end{array}$ & 50 & $\begin{array}{l}\text { Liraglutide } 0.6-1.8 \mathrm{mg} \text { daily versus } \\
\text { placebo }\end{array}$ & & March 2016 \\
\hline Exenatide once-weekly & EXSCEL (NCT01144338) & 14,000 & $\begin{array}{l}\text { Exenatide } 2 \text { mg weekly versus } \\
\text { placebo }\end{array}$ & & September 2017 \\
\hline Dulaglutide & REWIND (NCT01394952) & 9,622 & $\begin{array}{l}\text { Dulaglutide } 1.5 \text { mg weekly versus } \\
\text { placebo }\end{array}$ & & July 2018 \\
\hline Albiglutide & $\begin{array}{l}\text { HARMONY Outcomes } \\
\text { (NCT02465515) }\end{array}$ & 9,400 & $\begin{array}{l}\text { Albiglutide } 30 \text { mg or } 50 \text { mg weekly } \\
\text { versus placebo }\end{array}$ & & May 2019 \\
\hline Dapagliflozin & $\begin{array}{l}\text { DECLARE-TIMI58 } \\
\text { (NCT01730534) }\end{array}$ & 17,276 & $\begin{array}{l}\text { Dapagliflozin } 10 \text { mg daily versus } \\
\text { placebo }\end{array}$ & & April 2019 \\
\hline Ertugliflozin & VERTIS CV (NCT01986881) & 8,000 & $\begin{array}{l}\text { Ertugliflozin } 5 \text { mg or } 15 \text { mg daily } \\
\text { versus placebo }\end{array}$ & Drug not yet FDA or EMA approved & October 2019 \\
\hline \multirow[t]{2}{*}{ Linagliptin } & CAROLINA (NCT01243424) & 6,115 & $\begin{array}{l}\text { Linagliptin } 5 \text { mg daily versus } \\
\text { glimepiride 1-4 mg daily }\end{array}$ & & February 2019 \\
\hline & CARMELINA (NCT01897532) & 8,300 & $\begin{array}{l}\text { Linagliptin } 5 \text { mg daily versus } \\
\text { placebo }\end{array}$ & & January 2018 \\
\hline
\end{tabular}

$C V=$ cardiovascular; DPP-4 = dipeptidyl peptidase 4; EMA = European Medicines Agency; FDA = United States Food and Drug Administration; SFU = sulfonylurea.

Treated with ITCA 650 (FREEDOM-CVO) was completed in March 2016 and evaluated time to first occurrence of a composite endpoint of $\mathrm{CV}$ death, nonfatal $\mathrm{Ml}$, nonfatal stroke, or hospitalization for unstable angina for patients treated with investigational exenatide in DUROS ${ }^{\circledR}$ (Intarcia Therapeutics; ITCA 650) 60mcg daily. ${ }^{14}$ The drug ITCA 650 is a continuous subcutaneous delivery of exenatide via the DUROS device placed under the skin of the patient's abdomen twice yearly during an in-office procedure. Also completed in March 2016, the Effect of Liraglutide on Cardiovascular Endpoints in Diabetes Mellitus Type 2 Patients (MAGNA VICTORIA) aims to add data supporting the demonstrated CV benefit of liraglutide. ${ }^{15}$ The MAGNA VICTORIA investigators hypothesised that liraglutide improves CV function and has included targeted outcome measures that include stroke volume, ejection fraction and cardiac output. While both studies were completed in early 2016, results have not yet been published. The Exenatide Study of Cardiovascular Event Lowering (EXSCEL), the largest GLP-1 receptor agonist trial to date, recently reported out topline results indicating that EXSCEL met its primary safety endpoint of non-inferiority for MACE. ${ }^{16,17}$ Although there were fewer CV events in the exenatide arm of the trial, superior MACE reduction was not achieved. Further evaluation of trial data is ongoing and more data are to be presented at the European Association for the Study of Diabetes (EASD) annual meeting in September 2017. ${ }^{17}$ Researching Cardiovascular Events with a Weekly Incretin in Diabetes (REWIND) and A Long Term, Randomised, Double Blind, Placebo-controlled Study to Determine the Effect of Albiglutide, When Added to Standard Blood Glucose Lowering Therapies, on Major Cardiovascular Events in Patients With Type 2 Diabetes Mellitus (HARMONY Outcomes) trials are ongoing and are evaluating the time to first occurrence of a composite of CV death, nonfatal MI or nonfatal stroke in patients treated with dulaglutide and albiglutide, respectively.18,19 The other two trials are expected to be completed within the next 2 years.

Two ongoing trials of SGLT2 inhibitors also seek to evaluate CV outcomes. The Multicenter Trial to Evaluate the Effect of Dapagliflozin on the Incidence of Cardiovascular Events (DECLARE-TIMI58) and Cardiovascular Outcomes Following Ertugliflozin Treatment in Type 2 Diabetes Mellitus Participants With Vascular Disease (VERTIS CV) Study are each evaluating an SGLT2 inhibitor and time to first occurrence of CV death, nonfatal MI or nonfatal stroke. 20,21 DECLARE-TIMI58 is set to enroll over 17,000 patients and will be one of the largest CV outcomes trials to date. Ertugliflozin is not yet approved for use but the FDA and EMA both accepted new drug approval (NDA) filings for this agent in March 2017.

There are two ongoing CV outcomes studies of the DPP-4 inhibitor linagliptin. The Cardiovascular and Renal Microvascular Outcome Study with Linagliptin in Patients with Type 2 Diabetes Mellitus (CARMELINA) study is investigating the long-term impact of linagliptin versus placebo on CV morbidity and mortality (primary outcomes) and renal function (secondary outcomes) in patients with T2D at high risk of CV events. ${ }^{22}$ CARMELINA is a 48-month trial - two years longer than most other CV outcomes trials - with CV endpoints of time-to-firstoccurrence of a composite of CV death, nonfatal MI, nonfatal stroke and hospitalisation for unstable angina. The secondary renal endpoints include renal death, end-stage renal disease (ESRD), or a sustained decrease in eGFR of $50 \%$ or more. The Cardiovascular Outcome Study of Linagliptin Versus Glimepiride in Patients with Type 2 Diabetes (CAROLINA) trial is a comparison of CV outcomes a DPP-4 inhibitor (linagliptin) versus a sulfonylurea (glimepiride). ${ }^{23}$ CAROLINA is the largest trial of its kind comparing two oral antihypergylcemic agents head-to-head regarding their effects on CV outcomes. Given the concern for increased risk of HHF in the Saxagliptin Assessment of Vascular Outcomes Recorded in Patients with Diabetes Mellitus - Thrombolysis in Myocardial Infarction 53 (SAVOR-TIMI 53) trial, it will be important for the study investigators of these two linagliptin trials to report out on any HF-related findings. ${ }^{24}$

\section{Implications for practice}

Results of the published studies are influencing diabetes management guidelines; data from EMPA-REG OUTCOME and LEADER guided drug therapy recommendations in the 2017 American Diabetes Association Standards of Medical Care in Diabetes and the Canadian Pharmacologic Management of Type 2 Diabetes: November 2016 Interim Update. Both guidelines endorse the use of empagliflozin or liraglutide in patients with established CVD to reduce their risk of $\mathrm{CV}$ mortality. ${ }^{25-27}$ The positive $\mathrm{CV}$ outcomes data for semaglutide will likely be included in the next guideline update. It is unclear how the 
positive CV outcomes data for canagliflozin will be incorporated into updated guidelines and, ultimately, into practice given the increased risk of amputations and new black box warning related to this.

The mechanism(s) behind the CV benefit of empagliflozin, liraglutide, semaglutide and canagliflozin have not been clearly established. All four drugs have multiple beneficial effects on the body, such as glucose lowering, blood pressure (BP) lowering, and weight loss. It has been hypothesised that improvements in glycaemic control, BP and weight are linked with positive CV outcomes. However, the effects of empagliflozin, liraglutide, semaglutide and canagliflozin on these parameters in their respective CV outcomes trials were relatively modest. Across the three CV outcomes trials for the four drugs, A1C was reduced $0.24 \%$ to $0.7 \%$, systolic BP was reduced 1 to $4 \mathrm{mmHg}$, and weight was reduced 2 to $4 \mathrm{~kg}$ compared with placebo. ${ }^{9-11}$ While reduction in each surrogate marker is beneficial to the patient, the small magnitude of change does not explain the $\mathrm{CV}$ benefit. ${ }^{28-30}$ The beneficial $\mathrm{CV}$ effects of empagliflozin are possibly related to in the body's use of ketones as a fuel source. ${ }^{31}$ Empagliflozin may improve cardiac contractility and efficiency via preferential use of ketone bodies that produce energy more efficiently than glucose or free fatty acids. ${ }^{31}$ Because canagliflozin has the same mechanism of action as empagliflozin, this hypothesis is likely true for canagliflozin as well. The beneficial effects of liraglutide and semaglutide are possibly related to atherosclerosis prevention, though the definitive mechanism by which this occurs is unclear. ${ }^{10,28,32}$
Until more data are available, it is unknown whether the beneficial CV effects of these drugs represent a class effect (for SGLT-2 inhibitors and GLP-1 receptor agonists) or if the $\mathrm{CV}$ benefit is unique to the individual agents. Another unknown is whether these CV benefits extend to a lower CV risk population, such as primary prevention patients. Caution must be exercised to not extrapolate the results of EMPAREG OUTCOME, LEADER, SUSTAIN-6 and the CANVAS programme to all patients with T2D. However, both SGLT-2 inhibitors and GLP-1 receptor agonists are guideline-supported add-on therapies irrespective of a patient's CV risk. ${ }^{26}$

\section{Conclusion}

Canagliflozin, empagliflozin, liraglutide and semaglutide have all demonstrated CV benefit in patients with T2D and existing CVD and/or high risk of CVD. Given the risk of amputation, canagliflozin is the least preferred agent of the four despite its positive CV benefit. Preference should be given to the use of empagliflozin, liraglutide and semaglutide as add-on to first-line therapy in patients with T2D and CVD or at high CVD risk who require additional medication for glucose lowering. Caution should be exercised regarding potential adverse effects; not every patient is a candidate for these hyperglycaemic agents. Multiple other CV outcomes studies are underway and will provide more insights into additional agents that also have favourable CV effects, mechanisms of $\mathrm{CV}$ benefit, whether $\mathrm{CV}$ benefits are a class effect and whether lower-risk patients derive $\mathrm{CV}$ benefit from these agents. $\square$
1. Emerging Risk Factors Collaboration TERF, Sarwar N, Gao P, et al., Diabetes mellitus, fasting blood glucose concentration and risk of vascular disease: a collaborative meta-analysis of 102 prospective studies, Lancet, 2010;375:2215-22.

2. Gregg EW, Cheng YJ, Saydah S, et al., Trends in death rates among US adults with and without diabetes between 1997 and 2006: findings from the National Health Interview Survey Diabetes Care, 2012;35:1252-57.

3. Ferrannini E, DeFronzo RA, Impact of glucose-lowering drugs on cardiovascular disease in type 2 diabetes, Eur Heart J, 2015;36:2288-96.

4. Action to Control Cardiovascular Risk in Diabetes Study Group, Gerstein HC, Miller ME, et al., Effects of intensive glucose lowering in type 2 diabetes, N Eng/ J Med, 2008;358:2545-59.

5. Home PD, Pocock SJ, Beck-Nielsen H, et al., Rosiglitazone evaluated for cardiovascular outcomes in oral agent combination therapy for type 2 diabetes (RECORD): a multicentre, randomised, open-label trial, Lancet, 2009;373:2125-35.

6. Nissen SE, Wolski K, Effect of rosiglitazone on the risk of myocardial infarction and death from cardiovascular causes, N Engl J Med, 2007;356:2457-71

7. US FDA, Guidance for industry diabetes mellitus - Evaluating cardiovascular risk in new antidiabetic therapies to treat type 2 diabetes, 2008. Available at: www.fda.gov/cder/guidance/index. htm (accessed 30 March 2017).

8. EMA Guideline on the clinical investigation of medicinal products in the treatment or prevention of diabetes mellitus, Effective November 2012. Available at www.ema.europa.eu/ docs/en_GB/document_library/Scientific_guideline/2012/06/ docs/en_GB/document_library/Scientific__o
WC500129256.pdf (accessed 16 May 2017).

9. Zinman B, Wanner C, Lachin JM, et al., Empagliflozin, cardiovascular outcomes, and mortality in type 2 diabetes, N Engl I Med, 2015:373:2117-28.

10. Marso SP, Daniels GH, Brown-Frandsen $K$, et al., Liraglutide and cardiovascular outcomes in type 2 diabetes, N Engl J Med, 2016;375:311-22.

11. Marso SP, Bain SC, Consoli A, et al., Semaglutide and cardiovascular outcomes in patients with type 2 diabetes, N Engl J Med, 2016;375:1834-44.
12. Neal B, Perkovic V, Mahaffey, et al., Canagliflozin and cardiovascular and renal events in type 2 diabetes, N Eng/ J Med, 2017; doi: 10.1056/NEJMoa1611925. [Epub ahead of print]

13. Kosibrod M, Cavender M, Fu AZ, et al., Lower risk of heart. failure and death in patients initiated on SGLT-2 inhibitors versus other glucose-lowering drugs: The CVD-REAL Study, Circulation, 2017; doi: 10.1161/CIRCULATIONAHA.117.029190. [Epub ahead of print].

14. A study to evaluate cardiovascular outcomes in patients with type 2 diabetes treated with ITCA 650, 2017. Available at: http:// clinicaltrials.gov/ct2 (NCT01455896) (accessed 10 July 2017).

15. Effect of liraglutide on cardiovascular endpoints in diabetes mellitus type 2 patients (MAGNA VICTORIA), 2016 Availab at: http:///linicaltrials.gov/ct2 (NCT01761318) (accessed 10 at: http://cilic
July 2017).

16. Exenatide study of cardiovascular event lowering trial (EXSCEL): A trial to evaluate cardiovascular outcomes after treatment with exenatide once weekly in patients with type 2 diabetes mellitus, 2016. Available at: http://clinicaltrials.gov/ct2 (NCT01144338). (accessed 10 July 2017)

17. AstraZeneca, BYDUREON EXSCEL trial meets primary safety objective in type-2 diabetes patients at wide range of cardiovascular risk. Available at: www.astrazeneca-us.com/ media/press-releases/2017/bydureon-exscel-trial-meets -primary-safety-objective-in-type-2-diabetes-patients-at-wide -range-of-cardiovascular-risk-05232017.html (accessed 18 July 2017).

18. Researching cardiovascular events with a weekly incretin in diabetes (REWIND), 2016. Available at: http://clinicaltrials.gov/ ct2 (NCT01394952) (accessed 10 July 2017).

19. Effect of albiglutide, when added to standard blood glucose lowering therapies, on major cardiovascular events in subjects with type 2 diabetes mellitus, 2017. Available at: http://clinicaltrials.gov/ct2 (NCT02465515) (accessed 10 July 2017)

20. Multicenter trial to evaluate the effect of dapagliflozin on the incidence of cardiovascular events (DECLARE-TIMI58), 2017. Available at: http://clinicaltrials.gov/ct2 (NCT01730534) (accessed 10 July 2017)
21. Cardiovascular outcomes following ertugliflozin treatment in type 2 diabetes mellitus participants with vascular disease, The VERTIS CV Study (MK-8835-004), 2017. Available at: http://clinicaltrials.gov/ct2 (NCT01986881) (accessed 10 July 2017)

22. Cardiovascular and renal microvascular outcomes study with linagliptin in patients with type 2 diabetes mellitus (CARMELINA), 2017. Available at: http://clinicaltrials.gov/ct2 (NCT01897532) (accessed 16 May 2017).

23. CAROLINA: Cardiovascular outcome study of linagliptin versus glimepiride in patients with type 2 diabetes, 2017. Available at: http://clinicaltrials.gov/ct2 (NCT01243424) (accessed 16 May 2017).

24. Scirica BM, Bhatt DL, Braunwald E, et al. Saxagliptin and cardiovascular outcomes in patients with type 2 diabetes mellitus, N Eng/ I Med, 2013;369:1317-26.

25. Standards of medical care in diabetes-2017: Summary of revisions, Diabetes Care, 2016;40(Suppl 1):S4-S5.

26. American Diabetes Association, 8. Pharmacologic approaches to glycemic treatment, Diabetes Care, 2017;40(Suppl 1):S64S74.

27. Booth $G$, Lipscombe L, Butalia S, et al., Pharmacologic management of type 2 diabetes: 2016 interim update, Can J Diabetes, 2016;40:484-6.

28. Verges B, Charbonnel B, After the LEADER trial and SUSTAIN-6, how do we explain the cardiovascular benefits of some GLP-1 receptor agonists?, Diabetes Metab, 2017:43(Suppl 1): $2 S 3-2 S 12$.

29. Trujillo JM, Wettergreen SA, Nuffer WA, et al., Cardiovascular outcomes of new medications for type 2 diabetes, Diabetes Technol Ther, 2016:18:749-58.

30. Perseghin G, Solini A, The EMPA-REG outcome study: critical appraisal and potential clinical implications, Cardiovasc Diabetol, 2016:15:85.

31. Mudaliar S, Alloju S, Henry RR, Can a shift in fuel energetics explain the beneficial cardiorenal outcomes in the EMPA-REG OUTCOME study? A Unifying Hypothesis, Diabetes Care, 2016;39:1115-22

32. Ussher JR, Drucker DJ, Cardiovascular actions of incretin-based therapies, Circ Res, 2014;114:1788-803. 